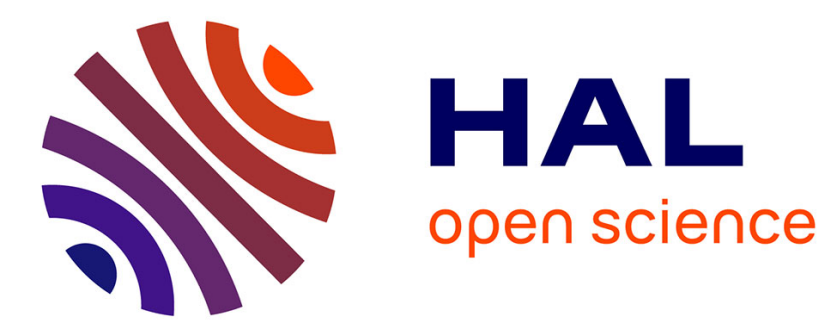

\title{
Etude de la rémanence de l'azinphos-méthyl utilisé contre Epiphyas postvittana Walker (Lepid.: Tortricidae) en Nouvelle-Zélande
}

David Maxwell Suckling

\section{- To cite this version:}

David Maxwell Suckling. Etude de la rémanence de l'azinphos-méthyl utilisé contre Epiphyas postvittana Walker (Lepid.: Tortricidae) en Nouvelle-Zélande. Agronomie, 1985, 5 (10), pp.893-898. hal00884718

\author{
HAL Id: hal-00884718 \\ https://hal.science/hal-00884718
}

Submitted on 1 Jan 1985

HAL is a multi-disciplinary open access archive for the deposit and dissemination of scientific research documents, whether they are published or not. The documents may come from teaching and research institutions in France or abroad, or from public or private research centers.
L'archive ouverte pluridisciplinaire HAL, est destinée au dépôt et à la diffusion de documents scientifiques de niveau recherche, publiés ou non, émanant des établissements d'enseignement et de recherche français ou étrangers, des laboratoires publics ou privés. 


\section{Etude de la rémanence de l'azinphos-méthyl uti- lisé contre Epiphyas postvittana Walker (Lepid. : Tortricidae) en Nouvelle-Zélande}

David Maxwell SUCKLING

Entomology Division, Department of Scientific and Industrial Research, Christchurch, New Zealand

RÉSUMÉ

La diminution des résidus d'azinphos-méthyl sur des feuilles de pommiers est étudiée en chromatographie en phase gazeuse à la suite de 3 traitements appliqués en verger. Parallèlement, la perte d'efficacité des résidus est étudiée par des essais biologiques effectués sur des larves d'Epiphyas postvittana. L'application de $0,9 \mathrm{~kg}$ de $\mathrm{m}$.a. /ha conduit à des résidus de $2-3 \mu \mathrm{g} / \mathrm{cm}^{2}$ de feuille. La diminution des résidus est plus rapide au printemps avec une réduction de moitié en $5,0-5,5 \mathrm{j}$, qu'en automne où la demi-vie des résidus est de $12,5 \mathrm{j}$. L'efficacité de l'azinphos-méthyl sur les larves diminue avec la réduction des résidus et les néonates sont plus sensibles que les larves d'âge moyen $\left(\mathrm{L}_{3}\right)$. La croissance des feuilles permet d'expliquer plus de $70 \mathrm{p} .100$ de la diminution des résidus entre le printemps et l'automne. Il est done possible de réduire la fréquence des traitements appliqués dans la lutte contre les tordeuses, particulièrement avant la récolte lorsque la croissance des feuilles est terminée.

Mots clés additionnels : Résidu, essai biologique, verger, pommier, organophosphoré.

The persistence of azinphos-methyl against Epiphyas postvittana Walker (Lepid. : Tortricidae) in New Zealand.

A study of the persistence of azinphos-methyl residues in relation to toxicity to lightbrown apple moth larval mortality showed that residues and insecticide efficacy declined in parallel during the 3 trials on 'Red Delicious' trees at Lincoln College. Gas chromatography of leaf discs punched at regular intervals showed that an application rate of $0.9 \mathrm{~kg} / \mathrm{ha}$ resulted in residues of $2-3 \mu \mathrm{g}$ azinphos-methyl $/ \mathrm{cm}^{2}$ of leaf area. Residues declined logarithmically, although this took place much faster in spring than in late summer. Two components of tree growth, leaf area and the number of leaves present on specific branches, were measured weekly. Both parameters increased during the spring, but levelled off after December. The decline in insecticide residues is thought to be increased by leaf growth during spring, by decreasing residues per unit area on sprayed growing foliage, as well as adding unsprayed foliage to the total leaf area present on the trees. Over $70 \%$ of the difference in rate of residue decline between seasons could be explained by the difference in leaf growth. Rainfall was an important second variable after time in multiple correlation analysis of residue decline, but day degrees and cumulative sunshine hours were unimportant. First instar lightbrown apple moth larvae were .more susceptible to azinphos-methyl residues than 3rd instar larvae. A comparison of results from laboratory treated and field sprayed foliage showed that 3rd instars provided better agreement between laboratory and field, possibly because of behavioural differences between larval stages.

Additional key words : Residue, bioassay, orchard, apple tree, organophosphate.

\section{INTRODUCTION}

La tordeuse des feuilles, Epiphyas postvittana (Walker), est un des 3 principaux ravageurs des arbres fruitiers de Nouvelle-Zélande. Elle s'attaque à plus de 125 genres de plantes (GEIER \& BRIESE, 1981) comprenant, entre autres, les pommiers, les framboisiers, les kiwis et les plantes ornementales. La biologie de cet insecte ressemble à celle de la tordeuse de la pelure
d'Europe, Adoxophyes orana (F.v.R.). Les fruits exportés de Nouvelle-Zélande doivent être garantis exempts de cet insecte. En Nouvelle-Zélande, 3 espèces de tortricides (les 2 autres sont Planotortrix excessana (Walker) et Ctenopseustis obliquana (Walker)) exercent une menace permanente qui rend la lutte chimique indispensable. Dans les vergers de pommiers, 9 à 10 traitements à l'azinphos-méthyl sont appliqués annuellement à intervalles de $14 \mathrm{j}$ contre ces espèces. 
Cependant, les données concernant la relation entre la dose et la mortalité, la résistance au lessivage et la rémanence de l'efficacité sur les larves sont encore insuffisantes. Il est par conséquent nécessaire d'étudier ici le rôle de la croissance des feuilles et de la pluviosité sur l'évolution de l'efficacité de l'insecticide comme CHARMILLOT \& BLASER (1983) l'ont fait pour A. orana.

\section{MATÉRIEL ET MÉTHODE}

\section{A. Analyse de l'azinphos-méthyl}

L'étude de la rémanence de l'azinphos-méthyl (50 p. 100 de m.a.) est effectuée sur des pommiers "Red Delicious » dans le verger d'essai à Lincoln College, Canterbury $\left(44^{\circ} \mathrm{S}, 172^{\circ} \mathrm{E}\right)$. L'analyse de résidus est réalisée par chromatographie en phase gazeuse à la suite des 3 traitements appliqués en novembredécembre 1981, janvier-février 1982 et novembredécembre 1982. Les applications sont effectuées le matin par l'arboriculteur au moyen d'un turbodiffuseur, à raison de 6001 et $900 \mathrm{~g}$ de m.a. par ha. Chaque prélèvement est opéré sur 4 arbres de la façon suivante ;

- 100 disques foliaires d'un diamètre de $20 \mathrm{~mm}$ sont pris au hasard entre 9 et $11 \mathrm{~h}$ du matin sur le quart Est de la couronne de l'arbre entre 1 et $2 \mathrm{~m}$ de hauteur.

- Pour les essais biologiques, 16 disques foliaires sont prélevés de la même façon sur les mêmes pommiers.

L'azinphos-méthyl est extrait de la surface foliaire par 3 lavages successifs (total $50 \mathrm{ml}$ ) au moyen d'un mélange d'éther et d'acétone dans la proportion 3:2. Un volume de $5 \mathrm{ml}$ de solution est conservé à - $15^{\circ} \mathrm{C}$ jusqu'à l'analyse chromatographique (Varian 3700) effectuée par un détecteur spécifique pour les produits organophosphorés (thermoionique). Le taux de récupération de l'azinphos-méthyl est déterminé par injection d'une solution standard; les résidus sont exprimés en $\mu \mathrm{g}$ d'azinphos-méthyl $/ \mathrm{cm}^{2}$ de feuille traitée. La chromatographie est effectuée dans les conditions suivantes :
Gaz:

$\mathrm{N}_{2}: 30 \mathrm{ml} / \mathrm{mn}$

Air : $175 \mathrm{ml} / \mathrm{mn}$

$\mathrm{H}_{2}: 4,5 \mathrm{ml} / \mathrm{mn}$
Température :

Détecteur : $230{ }^{\circ} \mathrm{C}$

Colonne : $220{ }^{\circ} \mathrm{C}$

Injecteur : $250^{\circ} \mathrm{C}$
La colonne mesure $1 \mathrm{~m}$ de longueur et $1,8 \mathrm{~mm}$ de diamètre intérieur.

La phase stationnaire est constituée par le support Gaschrom Q (100/120).

Pour les essais biologiques, 20 à 30 larves néonates de $E$. postvittana sont déposées dans chacune des 4 capsules de gélatine (numéro 000) avec 2 disques foliaires par capsule. Simultanément, 5 larves de stade moyen $\left(\mathrm{L}_{3}\right)$ sont déposées dans 4 autres capsules selon la même méthode. Les capsules sont mises en cellule climatisée à $20{ }^{\circ} \mathrm{C}$ et $70-80 \mathrm{p} .100$ d'humidité relative pour une durée de $16 \mathrm{~h}$, car la survie des larves dans le témoin est compromise après ce délai. Ensuite, le dénombrement des larves survivantes est réalisé à l'aide d'une loupe binoculaire. L'efficacité est calculée par rapport au taux de survie de larves déposées sur des feuilles témoins, prélevées à la même date sur un arbre non traité.

Pour confirmer les résultats obtenus sur feuilles traitées en verger, une autre méthode d'application de l'azinphos-méthyl est réalisée en laboratoire sur disques foliaires : les disques foliaires reçoivent sur chaque face pendant $10 \mathrm{~s}$ une pulvérisation de $2 \mathrm{ml}$ à la pression de 1 bar au moyen d'un tour de POTTER (PoTTER, 1952). Les résidus sont analysés (SUCKLING et al., 1984) et la même méthode d'essai biologique est appliquée sur des larves de stades $L_{1}$ et $L_{3}$. Pour chaque stade larvaire les essais sont répétés au moins 2 fois. Huit disques, 2 dans chaque capsule, sont utilisés avec 5 concentrations de produit, ainsi qu'un témoin non traité pour chaque répétition. Les analyses statistiques sont réalisées au moyen du programme POLO (ROBERTSON et al., 1980).

\section{B. Croissance des organes foliaires des arbres}

L'étude de l'effet de dilution consécutif à la croissance du feuillage s'est avérée nécessaire du fait que la réduction des résidus évoluait différemment entre les essais réalisés au printemps et à l'automne.

Sur chacun des 8 arbres situés de part et d'autre des 4 arbres utilisés pour l'étude de la rémanence, la croissance des feuilles est mesurée en prélevant 40 feuilles par arbre à intervalles réguliers d'une semaine (320 feuilles par semaine). Les mesures de surface foliaire sont effectuées à l'aide d'un appareil photo-électrique (Li-COR Modèle Li-3000). L'apparition de nouvelles feuilles est déterminée en comptant les feuilles sur une branche marquée, sur chacun des 8 arbres déjà mentionnés.

\section{Relevés météorologiques}

Les données concernant la pluviosité, la durée d'ensoleillement et le cumul en degrés-jours (dj) des sommes de températures supérieures à $0{ }^{\circ} \mathrm{C}$ sont relevées à la station météorologique du verger de Lincoln College.

\section{RÉSULTATS ET DISCUSSION}

\section{A. Rémanence de l'azinphos-méthyl (fig. 1)}

La méthode d'extraction décrite permet de récupérer plus de 90 p. 100 de l'azinphos-méthyl. La décroissance des résidus sur feuilles de pommiers est plus rapide au printemps (novembre-décembre) (fig. la et 1c) qu'en automne (fig. 1b). Les traitements du printemps sont les premiers de la saison (fig. la et 1c), alors que les résidus des traitements précédents s'ajoutent à ceux des traitements d'automne (fig. 1b). Des résultats similaires ont également été obtenus dans d'autres études (HALL et al., 1975; HAGLEY \& CHIBA, 1980). Le taux de diminution des résidus est logarithmique. La prise en considération des conditions météorologiques, par exemple des précipitations, ne permet pas d'améliorer significativement la description mathématique de la rémanence des résidus. 

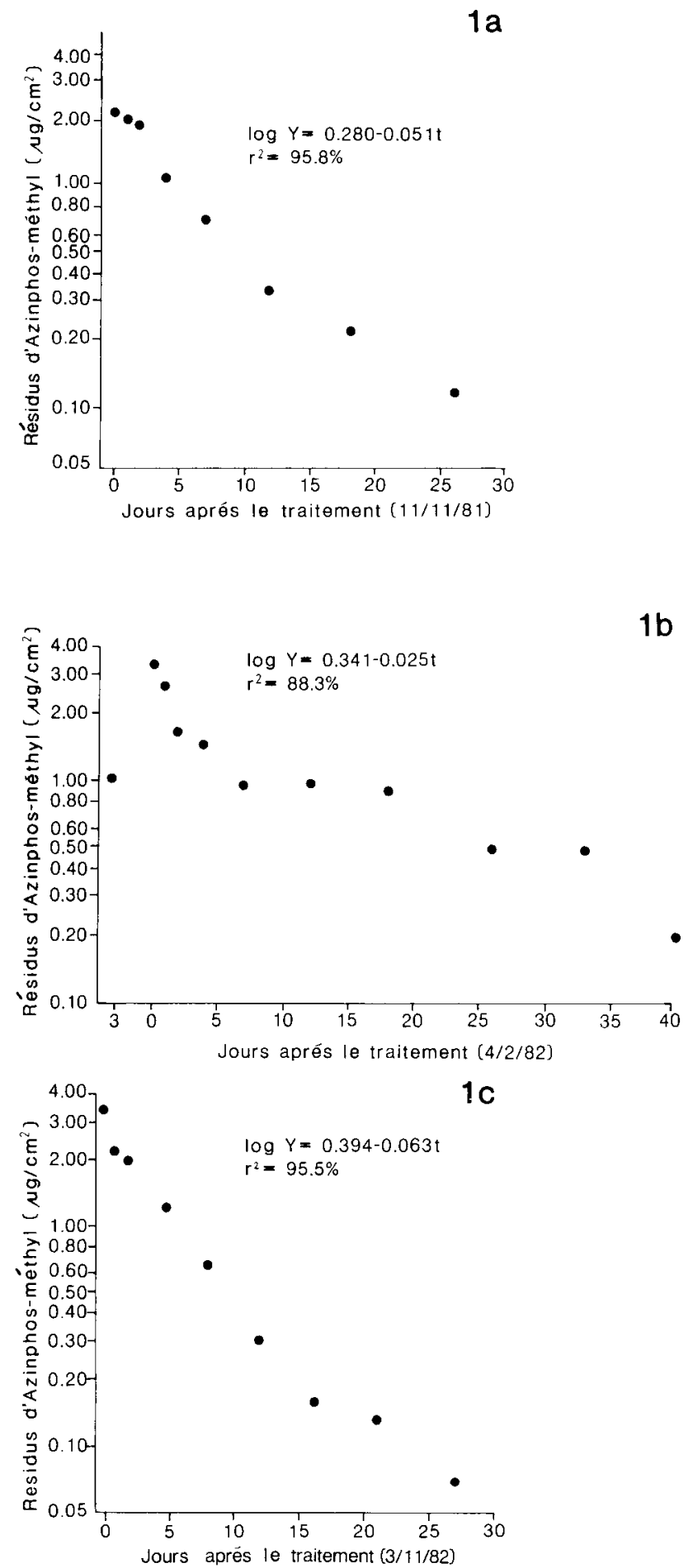

Figure 1

Diminution des résidus d'azinphos-méthyl sur des feuilles de pommier après des traitements effectués au printemps (la et 1c) ou en automne (lb).

Disappearance of residues of azinphos-methyl on apple leaves after treatments carried out in spring (la and $1 \mathrm{c}$ ) and autumn $(1 \mathrm{~b})$.

\section{B. Evolution de l'efficacité}

La décroissance des résidus d'azinphos-méthyl sur feuilles est accompagnée d'une perte d'efficacité sur E. postvittana (fig. 2). La mortalité dans les témoins des larves néonates est en moyenne de 4 à 8 p. 100 et elle est nulle chez les larves d'âge moyen. Signalons
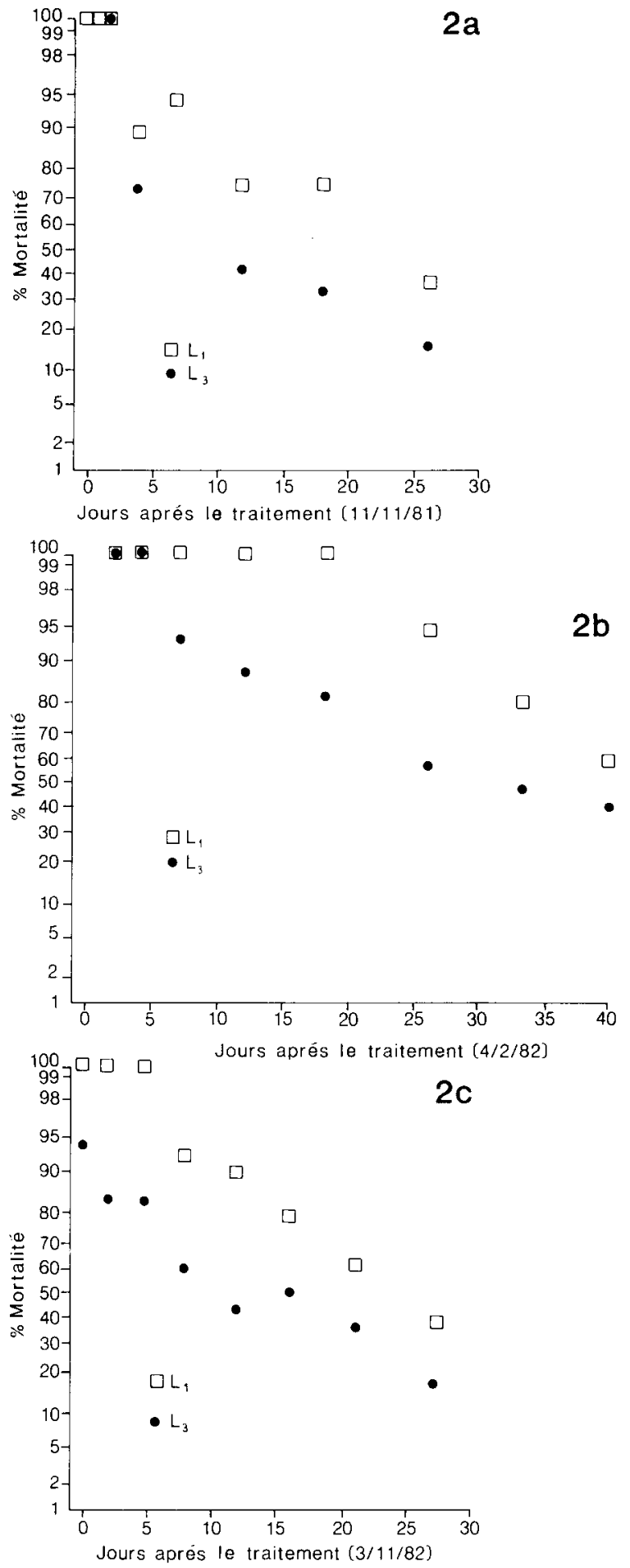

Figure 2

Evolution de l'efficacité de l'azinphos-méthyl sur des larves $d^{\prime} \mathrm{E}$. postvittana à la suite de traitements effectués au printemps ou en automne.

Change in efficacy of azinphos-methyl against $\mathrm{E}$. postvittana following treatments carried out in spring or in autumn.

que les taux de mortalité sont donnés en échelle probit sur les graphiques de la figure 2 . Après cette trañsformation, la mortalité régresse de façon linéaire en fonction du temps comme le log. des résidus. Les néonates sont plus sensibles à l'azinphos-méthyl que les larves de stade moyen $\left(\mathrm{L}_{3}\right)$. Le tableau 1 résume les résultats des essais biologiques. Durant les 2 essais 
TABLEAU

Comparaison des essais visant à déterminer la perte d'efficacité de l'azinphos-méthyl sur les larves $d$ 'E. postvittana.

Comparison of tests to determine the loss of efficacy of azinphos-methyl against larvae of $\mathrm{E}$. postvittana.

\begin{tabular}{|c|c|c|c|}
\hline & \multicolumn{2}{|c|}{ Essais biologiques } & $\begin{array}{c}\text { Essais } \\
\text { chimiques }\end{array}$ \\
\hline \multirow[t]{2}{*}{ Essai, date } & \multicolumn{2}{|c|}{$\begin{array}{c}\text { Durée après traitement jusqu'à } \\
\text { obtention de la } \mathrm{DL}_{50} \text { (jours) }\end{array}$} & \multirow{2}{*}{$\begin{array}{l}\text { Demi-vie des } \\
\text { résidus } \\
\text { (jours) }\end{array}$} \\
\hline & Larves néonates & Larves $\mathrm{L}_{3}$ & \\
\hline 13.11 .1981 & 21,5 & 14,0 & 5,5 \\
\hline 311.11 .1982 & 22,5 & 13,4 & 5,0 \\
\hline 2 3. 2.1982 & 45,0 & 33,8 & 12,5 \\
\hline
\end{tabular}

effectués en novembre, les décroissances des résidus sont comparables. Dans les 2 cas, nous constatons une diminution de moitié après environ $5 \mathrm{j}$. En février, par contre, il faut attendre plus de $12 \mathrm{j}$ pour obtenir une décroissance de moitié des résidus. Parallèlement, les essais biologiques montrent que le taux de mortalité tombe à 50 p. 100 au cours du laps de temps qui correspond à une perte de moitié des résidus. Cependant, la mortalité totale n'est probablement pas encore atteinte après la durée d'exposition des larves de $16 \mathrm{~h}$ (SUCKLING, 1983).

Les analyses en probit des essais sur l'efficacité de l'azinphos-méthyl avec disques foliaires traités en laboratoire ou en verger sont similaires (tabl. 2). L'analyse chimique des résidus sur pommiers lors des différents prélèvements a donné des résultats conformes à la mortalité obtenue sur les larves aux dates correspondantes, et ceci pour tous les essais.

Cependant, sur larves néonates, les essais montrent que les résidus sur feuilles traitées prélevées en verger et provoquant 50 p. 100 de mortalité (DL 50) sont supérieurs à ceux qui, sur des feuilles traitées en laboratoire, déterminent la DL 50. Bien que l'analyse ne permette pas de différencier les résidus déposés sur chacune des faces de la feuille, il faut admettre qu'en laboratoire les résidus obtenus à la tour de POTTER sont identiques sur les 2 faces de la feuille tandis qu'en verger, les résidus déposés sont plus faibles sur la face inférieure des feuilles (COOKE et al., 1975;
1976). Il faut ajouter qu'en verger les larves néonates colonisent toujours la face inférieure des feuilles et, par conséquent, celle sur laquelle les résidus sont les moins importants; ces 2 facteurs sont vraisemblablement les causes de la différence observée chez les larves néonates entre les traitements effectués en champ et en laboratoire.

Par contre, pour les larves d'âge moyen $\left(L_{3}\right)$ qui se développent indifféremment sur les 2 faces des feuilles, les essais effectués en verger et en laboratoire conduisent aux mêmes résultats (tabl. 2).

\section{Croissance des feuilles (fig. 3)}

Les diminutions des résidus étant très différentes entre le $1^{\text {er }}$ et le $2^{\mathrm{e}}$ essai, nous avons profité du $3^{\mathrm{e}}$ essai pour suivre l'évolution de la croissance du feuillage. La figure 3 montre que la croissance des feuilles s'arrête en décembre ralentissant ainsi la régression des résidus. Il est probable que la croissance des feuilles avait suivi le même schéma l'année précédente et qu'elle était terminée en février 1982 durant le $2^{\mathrm{e}}$ essai. Or, dans cet essai, 51 p. 100 des résidus présents $2 \mathrm{j}$ après le traitement sont encore dosables $12 \mathrm{j}$ plus tard (fig. 1b). Par contre, la figure 1c montre qu'avec la croissance, 18 p. 100 seulement des résidus présents $2 \mathrm{j}$ après le traitement peuvent être retrouvés $12 \mathrm{j}$ plus tard. Avec la croissance du végétal, le déclin des résidus est donc 2,9 fois plus important. Durant ce laps de temps, "l'Index de Croissance des Feuilles " (ICF), qui est le produit du nombre de feuilles par leur surface, s'élève à 2,1 . La croissance des feuilles peut donc expliquer plus de 70 p. 100 de la différence entre les diminutions de résidus d'azinphosméthyl observées entre le printemps et l'automne. Il en résulte que la croissance des feuilles est un facteur très important à considérer pour interpréter l'efficacité d'un traitement chimique contre les ravageurs des pommiers.

\section{Relevés météorologiques}

Les sommes de température et les durées d'ensoleilment sont plus importantes en automne qu'au printemps (tabl. 3). La pluviométrie par contre est plus

TABLEAU 2

Essais biologiques. Comparaison des 3 traitements à l'azinphos-méthyl effectués en verger avec le traitement en laboratoire. Bioassays. Comparison of 3 orchard sprays of azinphos-methyl with laboratory experiments.

\begin{tabular}{|c|c|c|c|c|c|c|}
\hline \multirow[b]{2}{*}{ Essai, date } & \multirow{3}{*}{$\frac{N}{4937}$} & \multicolumn{2}{|c|}{$\mathrm{L}_{1}$ larves néonates } & \multirow{3}{*}{$\frac{N}{256}$} & \multicolumn{2}{|c|}{$\mathrm{L}_{3}$ larves d'âge moyen } \\
\hline & & $\mathrm{DL}_{50}$ & Pente \pm e.t. & & $\mathrm{DL}_{50}$ & Pente \pm e.t \\
\hline 3.11 .1981 & & $0,15 \mu \mathrm{g} / \mathrm{cm}^{2}$ & $1,85 \pm 0,07$ & & $0,37 \mu \mathrm{g} / \mathrm{cm}^{2}$ & $2,03 \pm 0,31$ \\
\hline $2 \quad 3.2 .1982$ & 2780 & $0,17 \mu \mathrm{g} / \mathrm{cm}^{2}$ & $2,22 \pm 0,13$ & 384 & $0,32 \mu \mathrm{g} / \mathrm{cm}^{2}$ & $2,11 \pm 0,28$ \\
\hline $3 \quad 11.11 .1982$ & 3267 & $0,10 \mu \mathrm{g} / \mathrm{cm}^{2}$ & $2,09 \pm 0,09$ & 640 & $0,36 \mu \mathrm{g} / \mathrm{cm}^{2}$ & $1,32 \pm 0,12$ \\
\hline \multicolumn{7}{|c|}{ Essai en laboratoire } \\
\hline & 4046 & $0,05 \mu \mathrm{g} / \mathrm{cm}^{2}$ & $2,76 \pm 0,09$ & 164 & $0,30 \mu \mathrm{g} / \mathrm{cm}^{2}$ & $2,21 \pm 0,35$ \\
\hline
\end{tabular}

$\mathrm{N}$ : nombre d'insectes dans le test ; number of insects in the test.

$\mathrm{DL}_{50}$ : résidus causant une mortalité de $50 \mathrm{p}$. 100 ; résidues causing $50 \%$ mortality.

Pente : pente de la régression en probit; slope of the probit regression line.

e.t. : écart-type ; standard error. 


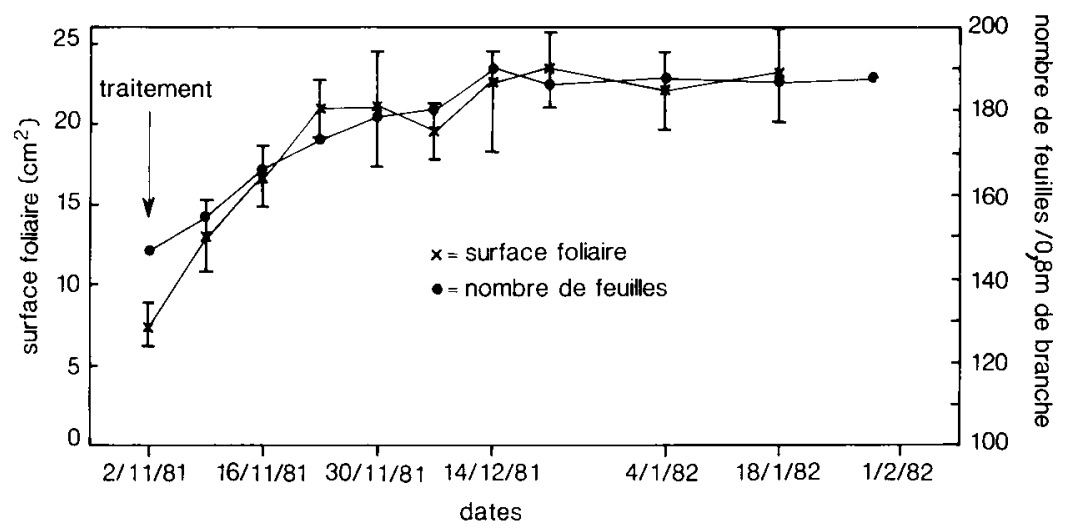

Figure 3

Evolution de la croissance des feuilles de pommier au cours du printemps.
Growth of apple leaves during spring.
TABLEAU 3

Relevés météorologiques au cours des 3 essais. Meteorological data from the 3 trials

\begin{tabular}{|c|c|c|c|c|}
\hline Essai & Jours & $\begin{array}{c}\text { Degrés/ } \\
\text { Jours }\end{array}$ & $\begin{array}{l}\text { Ensoleillement } \\
\text { cumulé (en h) }\end{array}$ & $\begin{array}{l}\text { Précipitations } \\
\text { cumulées }(\mathrm{mm})\end{array}$ \\
\hline
\end{tabular}

1. 11.11.1981 (Printemps)

$\begin{array}{rrrr}0 & 0 & 0 & 0 \\ 1 & 14 & 9 & 0 \\ 2 & 31 & 10 & 0,3 \\ 4 & 62 & 26 & 5,1 \\ 7 & 105 & 35 & 16,3 \\ 12 & 170 & 77 & 22,8 \\ 18 & 258 & 131 & 22,8 \\ 26 & 398 & 182 & 31,6\end{array}$

2. 4.2 .1982 (Automne)

$\begin{array}{rrrl}0 & 0 & 0 & 0 \\ 1 & 20 & 8 & 0 \\ 2 & 30 & 19 & 4,0 \\ 4 & 65 & 40 & 4,0 \\ 7 & 121 & 72 & 4,0 \\ 12 & 207 & 121 & 4,0 \\ 18 & 309 & 169 & 4,0 \\ 26 & 460 & 197 & 17,4 \\ 33 & 577 & 252 & 17,4 \\ 40 & 692 & 286 & 21,4\end{array}$

3. 3.11 .1982 (Printemps)

\begin{tabular}{rrrr}
0 & 0 & 0 & 0 \\
1 & 15 & 8 & 0 \\
2 & 27 & 16 & 0 \\
5 & 76 & 40 & 0 \\
8 & 126 & 55 & 0 \\
12 & 184 & 61 & 23,9 \\
16 & 243 & 91 & 23,9 \\
21 & 313 & 116 & 27,1 \\
27 & 401 & 164 & 55,0 \\
\hline
\end{tabular}

grande au printemps, cependant elle n'affecte pas la diminution des résidus d'azinphos-méthyl (SUCKLING, 1983).

\section{CONCLUSION}

Si l'on connaît le rapport existant entre l'insecte et l'insecticide ainsi que les facteurs de l'environnement qui affectent cette relation, il est alors possible de limiter le nombre des traitements insecticides effectués contre les ravageurs des pommiers. Pour des raisons de coût, de salubrité publique et de protection de l'environnement, il est souhaitable de limiter le nombre de traitements pour retarder ou éviter l'apparition de phénomènes de résistance (SUCKLING, 1983). Malheureusement dans les conditions de la NouvelleZélande, avec 3 espèces de tordeuses nuisibles aux cultures de pommiers qui exercent une menace permanente, il n'est pas possible de faire coïncider toutes les applications insecticides indispensables pour combattre ces ravageurs. D'autre part, du fait que les fruits destinés à l'exportation doivent être garantis exempts de ravageurs, la lutte est forcément intensive. Malgré cela, il semble possible de réduire tout de même la fréquence des traitements.

Les essais décrits ci-dessus montrent que la diminution des résidus d'azinphos-méthyl diffère de manière significative entre le printemps et l'automne. La mortalité des larves suit de près l'évolution des résidus, de sorte que la rémanence des traitements est plus importante en automne. La croissance du végétal permet d'expliquer cette différence. De même que la tordeuse de la pelure $A$. orana, $E$. postvittana attaque les pousses en croissance; il survient une perte d'efficacité causée par l'apparition et la croissance des feuilles (CHARMILlOT \& BLASER, 1983). Les arboriculteurs peuvent donc espacer les traitements dirigés contre les tordeuses dès la fin de la croissance de la végétation : cette réduction de la fréquence des interventions est d'autant plus réalisable que les fruits sont destinés à la commercialisation locale.

Recu le 16 avril 1985. Accepté le 8 juillet 1985.

\section{REMERCIEMENTS}

Je remercie le Dr D. R. PENMAN et M. R. B. CHAPMAN (Dept of Entomology, Lincoln College) pour leurs conseils ainsi que le Dr P. T. HOLLAND (Ruakura Soil and Plant Research Station) pour sa méthode analytique par chromatographie en phase gazeuse. Mes sincères remerciements s'adressent aussi à M. D. J. ROGERS et à Mlle K. FERGUSON pour leur assistance dans les essais ainsi qu'au DSIR pour le contrat de recherche no UV/1/30. Le milieu artificiel d'élevage a été fourni par Insect Rearing Section, Entomology Division. Je remercie enfin le Dr P. J. CHARMILLOT (Station Fédérale de Recherches Agronomiques de Changins, Suisse) pour son assistance dans la rédaction de cet article. 


\section{RÉFÉRENCES BIBLIOGRAPHIQUES}

Charmillot P. J., Blaser Ch., 1983. Etude de la rémanence de l'acéphate, du phosmet et de la deltaméthrine utilisés dans la lutte contre la tordeuse de la pelure Adoxophyes orana F.V.R. Rev. suisse Vitic. Arboric. Hortic., 15, 195-201.

Cooke B. K., Herrington P. J., Jones K. G., Morgan N. G., 1975 Spray deposit cover and fungicide distribution on intensive apple trees by over head mobile spraying methods. Pestic. Sci., 6, 581587.

Cook B. K., Herrington P. J., Jones K. G., Morgan N. G., 1976 Spray deposit cover and fungicide distribution obtained by low and ultra low volumes spraying of intensive apple trees. Pestic. Sci., 71, $35-40$.

Geier P. W., Briese D. T., 1981. The lightbrown apple moth Epiphyas postvittana (Walker). A native leafroller fostered by European settlement, p. 131-155. In Kitching R. L., Jones R. E. (eds) : «The ecology of pests : Some Australian case histories. Melbourne, CSIRO, 245 p.

Hagley E. A. C., Chiba M., 1980. Efficacy of phosmet and azinphos-methyl for control of insect pests of apple in Ontario. Can Entomol., 112, 1075-1083.
Hall F. R., Reichard D. L., Krueger H. R., 1975. Dislodgeable azinphos-methyl residues from air blast spraying of apple foliage in Ohio. Arch. environ. Contam. Toxicol., 3, 352-363.

Potter C., 1952. An improved laboratory apparatus for applying direct sprays and surface films, with data on the electrostatic charge on atomized spray fluids. Ann. appl. Biol., 39, 1-28.

Robertson J. L., Russell R. M., Savin N. E., 1980. POLO : A users guide to Probit or Logit Analysis. (A computer programme for insecticide bioassay data analysis). Berkeley Pacific South-West Forestry and Range Experiment Station, 14 p.

Suckling D. M., 1983. The responses of Epiphyas postvittana ( $\mathrm{Wal}$ ker) and Planotortrix excessana (Walker) (Lepidopters : Tortricidae) to insecticides. Ph. D. Thesis, Lincoln College, University of Canterbury, NZ., 197 p.

Suckling D. M., Chapman R. B., Penman D. R., 1984. Insecticide resistance in lightbrown apple moth : Larval responses to azinphosmethyl. J. econ. Entomol., 77, 579-582. 